\title{
The COVID-19 pandemic: is our medicine still evidence-based?
}

\author{
Cristian Deana ${ }^{1}$
}

Received: 5 May 2020 / Accepted: 11 May 2020 / Published online: 28 May 2020

(C) Royal Academy of Medicine in Ireland 2020

\begin{abstract}
There is no randomized controlled trial that demonstrated the efficacy of antiviral therapy against COVID-19 yet. However, physicians are prescribing different drugs to a large part of COVID-19 population in the hope they will cure them. This does not reflect the evidence-based medicine approach. What we need is more evidence-based knowledge about what routine care practices we should to apply to ameliorate symptoms of patients and fight COVID-19 pathology.
\end{abstract}

Keywords COVID-19 $\cdot$ Evidence-based medicine $\cdot$ Randomized controlled trial $\cdot$ Remdesivir

Since January 2020, an estimated 3 million people worldwide have become infected with severe acute respiratory syndrome coronavirus 2 (SARS-CoV-2) - the infectious agent causing the coronavirus disease-2019 (COVID-19) pandemic. Approximately 200,000 have died to date as a result of COVID-19 [1].

Every day, physicians have to make timely decisions about the best way to treat COVID-19 patients, and in the most severe cases, decisions may need to be taken extremely rapidly. In modern medicine, decision-making about the care of individual patients is based on the so-called "evidence-based medicine" (EBM) model, entailing the meticulous, judicious, explicit, and reasonable use of up-to-date best evidence [2].

The most robust scientific evidence regarding the identification of best treatments for a disease comes from randomized trials or metanalysis in which the possibility for bias is minimized; the next level (i.e., of a lower grade) is provided by cohort or case-control studies.

With the advent of COVID-19, a paradox is appearing: despite the large mass of potential patients eligible for studies, science has not been able to keep up with the vast volume of available raw data. The number of publications concerning COVID-19 that have appeared in PubMed since January 2020 exceed a staggering 7300 [3]. Moreover, as shown in Fig. 1, the number of registered studies on COVID-19 is growing linearly day-by-day with more than 900 to date [4].

Cristian Deana

deana.cristian@gmail.com

1 Anesthesia and Intensive Care 1, Department of Anesthesia and Intensive Care, Academic Hospital of Udine, Udine, Italy
At the same time, the number of infected people has been rising steeply since mid-March. But how many of the published studies provide sound, unbiased scientific evidence? How many report data and arrive at conclusions that help doctors apply EBM? To the best of our knowledge, only four RCTs have been performed and published until now, but none have the game-changing results we would have hoped for [5]. The probable reason is that RCT take too much time to complete and generate results, and time is not at our disposal during COVID-19. What is more, to be of high value, a RCT investigating the efficacy of a drug treatment should ideally compare the results to a placebo. But would this be ethical in the case of COVID-19? No simple answer ever exists to a complicated question. In fact, only one of the four RCTs included a placebo group when assessing the effects of a drug treatment (remdesivir was the medication under study). The other studies assessed the effects of remdesivir compared with other drugs such as lopinavir-ritonavir [6]. Whatever the approach taken, the COVID-19 pandemic offers clinicians the potential for patient recruitment as never seen before.

So it seems that the COVID-19 pandemic has changed the way of doing medicine: we are presently moving away from EBM towards a more observational approach. In doing so, are we laying the foundations for a new design of study that will perhaps lead us to the evidence we seek? We hope not. In fact, the only glimmer of hope obtained so far has come from the ad interim analysis of a well-designed ongoing RCT about remdesivir [7].

But the devil has not been hunted down yet, and COVID-19 patients require treatment. So what do we do in the meantime given the lack of evidence? The answer may lie in the words offered by the "Father of medicine"-Hippocrates-approximately 
Fig. 1 Shows the number of studies $(\Delta)$ registered by the WHO Trial Registry Network (available at https://clinicaltrials. gov/ct2/who_table) and the number of publications $(\mathrm{O})$ found at https://www.ncbi.nlm.nih.gov/ January to 21st April 2020 pubmed/?term=covid-19 from

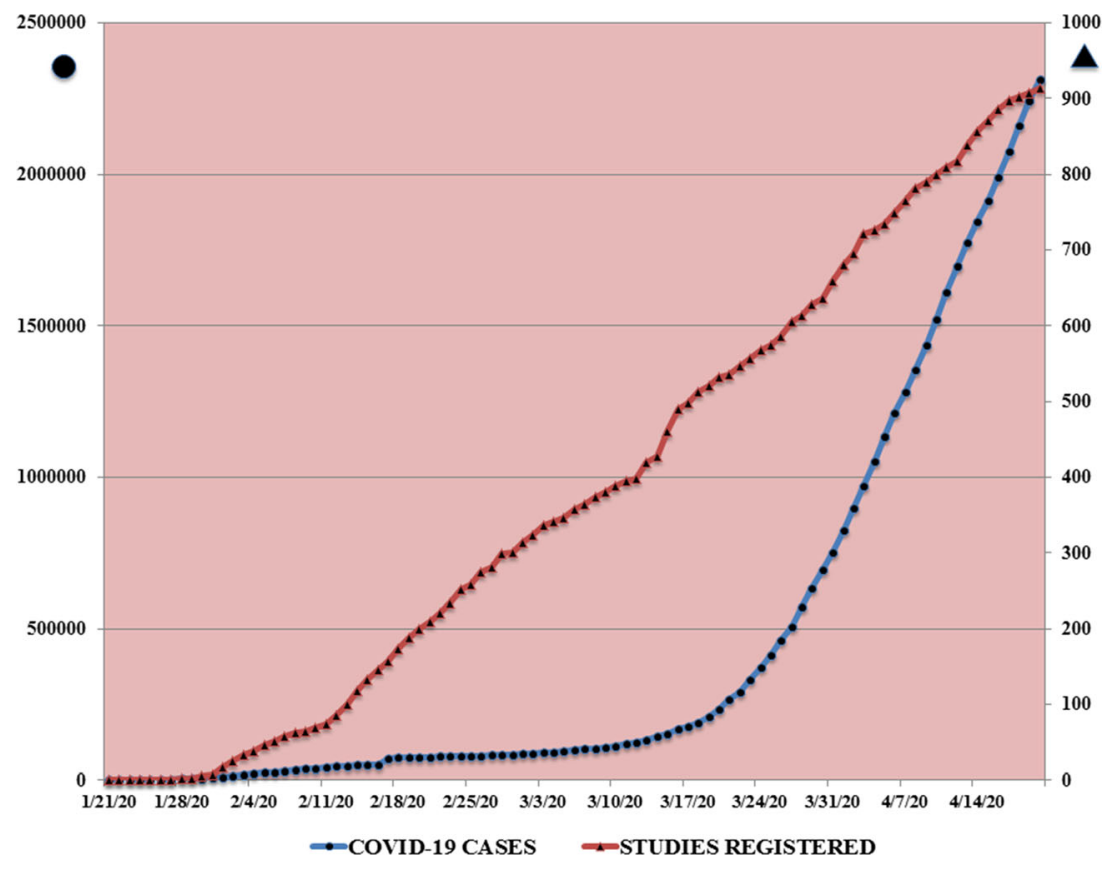

\section{References}

2500 years ago, who wrote "Primum non nocere, deinde curare," which translates into "first do no harm, and only then cure."

But despair does not authorize us to do anything! And we should not believe that doing something is necessarily better than doing nothing. Indeed, all drugs have their side effects. So, considering that the pathophysiology of COVID-19 has only just been elucidated, the results of the abovementioned RCTs that evaluated the treatment options identified and used until now could be misleading.

So perhaps this present "frenzy to publish" in relation to COVID-19 should be abandoned and downright avoided. To improve the outcome of critically ill patients, what we need is more evidence-based knowledge about what routine care practices we should to apply to ameliorate their symptoms and fight COVID-19 pathology. Doctors should return to the key principles of basic care and EBM. This is what COVID-19 patients need, and we owe it to them!

\section{Compliance with ethical standards}

Conflict of interest The author declares that he has no conflict of interest.
1. World Health Organization website. https://www.who.int/ (accessed $29^{\text {th }}$ April 2020)

2. Masic I, Miokovic M, Muhamedagic B (2008) Evidence based medicine - new approaches and challenges. Acta Inform Med 16(4):219 225

3. https://www.ncbi.nlm.nih.gov/pubmed/?term=covid-19 (accessed $29^{\text {th }}$ April 2020)

4. www.clinicaltrial.gov, (acessed on $29^{\text {th }}$ April 2020)

5. Thorlund K, Dron L, Park J, et al. A real-time dashboard of clinical trials for COVID-19. Lancet Published: April 24, 2020 https://doi. org/10.1016/S2589-7500(20)30086-8

6. Wang Y, Zhang D, Du G et al (2020) Remdesivir in adults with severe COVID-19: a randomised, double-blind, placebo-controlled, multicentre trial. Lancet. https://doi.org/10.1016/S0140-6736(20) 31022-9

7. https://www.niaid.nih.gov/news-events/nih-clinical-trial-showsremdesivir-accelerates-recovery-advanced-covid-19 (accessed $29^{\text {th }}$ April 2020)

Publisher's note Springer Nature remains neutral with regard to jurisdictional claims in published maps and institutional affiliations. 